\title{
Innovaciones estadísticas y redes neuronales artificiales aplicadas a la prospección geoquímica en los cuadrángulos de Chaparra y Atico, Caravelí - Arequipa
}

\author{
Statistical innovations and artificial neural networks applied to geochemical \\ prospection in Chaparra and Atico quadrangles, Caraveli - Arequipa
}

\author{
Orlando Bazán Santa Cruz
}

Recibido: 01/12/2020 - Aprobado: 12/19/2021 - Publicado: 23/12/2021

\begin{abstract}
RESUMEN
La prospección geoquímica siempre ha sido y seguirá siendo una de las herramientas más efectivas para la búsqueda de recursos minerales, que se integra con la cartografía geológica base y otras disciplinas como la teledetección y la geofísica. Esta investigación pretende impulsar una nueva metodología de prospección geoquímica combinando el índice de enriquecimiento relativo local (LREI) de elementos indicadores de mineralización ( $\mathrm{Cu}, \mathrm{Au}, \mathrm{Ag}, \mathrm{Pb}, \mathrm{Zn}$ y Mo), coeficientes de rango correlacional (RCC) y el análisis de componentes principales (PCA), a través de superposiciones difusas (fuzzy modelling) y el uso de aprendizaje profundo (deep learning) no supervisado, para generar mapas de prospectividad geoquímica en los cuadrángulos de Chaparra (320) y Atico (330) en la provincia de Caravelí, Arequipa. El mapa de prospectividad generado mediante superposición difusa y el mapa autoorganizado (SOM) fueron validados ploteando la ubicación de los depósitos y ocurrencias minerales conocidos, los cuales en la mayoría de los casos se plotean dentro de áreas con alta o moderada prospectividad. Por lo que se concluye que las innovaciones estadísticas univariables, multivariables e innovaciones tecnológicas aplicadas a la cartografía geoquímica son herramientas efectivas para la prospección geoquímica.
\end{abstract}

Palabras claves: Aprendizaje profundo; geoquímica; prospección; redes neuronales; superposición difusa.

\begin{abstract}
Geochemical prospecting has always been and will be one of the most effective tools for the mineral resources exploration, that integrates with the base geological mapping and other disciplines like remote sensing and geophysics. This investigation aim is to promote a new geochemical prospecting methodology, which combines the local relative enrichment index (LREI) from mineralization indicator elements ( $\mathrm{Cu}, \mathrm{Au}, \mathrm{Ag}, \mathrm{Pb}, \mathrm{Zn}$ and $\mathrm{Mo}$ ), range correlation coefficients (RCC) and Principal Components Analysis (PCA), through fuzzy overlays and unsupervised deep learning, to generate geochemical prospectivity maps in the Chaparra (320) and Atico (330) quadrangles in the province of Caraveli, Arequipa. The prospectivity map generated by fuzzy modelling and the self-organizing map (SOM) were validated plotting the location of the known deposits and mineralized occurrences, which in most of the cases are plotted within areas with high or moderate prospectivity. Therefore, it is concluded that univariate and multivariate statistical innovations and technological innovations applied to geochemical mapping are effective tools for geochemical prospection.
\end{abstract}

Keywords: Deep learning; geochemistry; prospecting; neural networks; fuzzy overlay.

\footnotetext{
1 Universidad Nacional Mayor de San Marcos, Unidad de Posgrado, Lima, Perú. Estudiante de la Maestría en Geología, mención en Recursos Mineros. Autor por correspondencia: orlando.bazan@unmsm.edu.pe ORCID: https://orcid.org/0000-0002-7764-9460
} 


\section{INTRODUCCIÓN}

Hoy en día, en la exploración geológica, se conoce una serie de innovaciones en el tratamiento estadístico de los datos geoquímicos, que están relegando al método tradicional de identificar parámetros geoquímicos (valor de fondo, umbral y anomalías) basado en la media y los múltiplos de la desviación estándar (HAWKES \& WEBB, 1963), así por ejemplo, tenemos los modelos fractales / multifractales (Cheng, 2007; Zuo \& Wang, 2016), estadísticas robustas de vecindario local (Zuo, 2014), el índice de enriquecimiento relativo local (LREI, por sus siglas en inglés) (Zuo, 2014), el gap estadístico local (Wang \& Zuo, 2016); la mayoría de estos métodos tienen en cuenta la variación del valor de fondo por medio de una técnica de ventana deslizante (Zhang et al., 2007; Wang \& Zuo, 2016). Asimismo, las estadísticas multivariables como el coeficiente de rango correlacional, análisis factorial (Tripathi, 1979; Reimann et al., 2002; Liu et al., 2016), el análisis de componentes principales (Zuo, 2011; Cheng et al., 2011; Zuo et al., 2013; Chen et al., 2019) se ha convertido en una poderosa herramienta de prospección basado en las asociaciones geoquímicas de los depósitos minerales. Estas técnicas de estadísticas univariables y multivariables pueden usarse de forma integrada a través de inferencias difusas (fuzzy modelling) (Ziaii et al., 2009; Yousefi et al., 2014; Moradi et al., 2015) para generar mapas de prospectividad a nivel regional y local. Con el desarrollo de la inteligencia artificial, aprendizaje automático (machine learning) y aprendizaje profundo (deep learning) surgen nuevas formas de integrar una gran cantidad de datos geoquímicos y usarlos en la cartografía geoquímica para localizar nuevos targets de exploración (Kirkwood et al., 2016; Zuo, 2017; Zuo \& Xiong, 2018; Zuo et al., 2019).

En los cuadrángulos de Chaparra y Atico en la provincia de Caravelí, Arequipa se cuenta con una basta información geoquímica generada por el INGEMMET en el año 2016, sin embargo, esta información no ha sido analizada e interpretada con rigurosidad, con un adecuado enfoque estadístico, a fin de maximizar su valor para la exploración geoquímica en dicha región.

Esta investigación pretende impulsar una nueva metodología de prospección geoquímica combinando el índice de enriquecimiento relativo local (LREI) de elementos indicadores de mineralización, el coeficiente de rango correlacional y el análisis de componentes principales a través de superposiciones difusas (fuzzy modelling) y el uso de aprendizaje profundo (deep learning) no supervisado, para generar mapas de prospectividad geoquímica en los cuadrángulos de Chaparra y Atico en la provincia de Caravelí, Arequipa; lo que permitirá vectorizar la exploración minera hacia las zonas con mayor probabilidad de encontrar nuevos depósitos minerales.

\section{METODOS}

\section{1. Área de estudio y data}

El área de estudio comprende los cuadrángulos de Chaparra (32o) y Atico (33o) de la carta geológica nacional, ubicados en la provincia de Caravelí al norte de la región Arequipa (Figura 1). La geología dentro del área consiste en secuencias clásticas, volcanoclásticas y carbonatadas, cuyas edades van del Paleozoico hasta el Cenozoico (Torres et al., 2017). La mineralización en el área de estudio está asociada a la intrusión de grandes volúmenes de material magmático durante el periodo Jurásico - Cretácico. La mayor parte de las ocurrencias metálicas están asociadas a vetas de cuarzo, óxidos y panizo; pero existen también evidencias de mineralización asociada a depósitos del tipo óxidos de hierro, cobre y oro (IOCG, por sus siglas en inglés), Pórfido de cobre y epitermales (Torres et al., 2017).

Para esta investigación se usan datos geoquímicos de sedimentos de corriente de las muestras recolectadas por el Instituto Geológico, Minero y Metalúrgico (INGEMMET) en los cuadrángulos de Chaparra y Atico en el año 2016, en el marco de cooperación e intercambio de información científica y técnica entre China Geological Survey y el INGEMMET, donde se recolectaron 3146 muestras de sedimento de corriente (Figura 1), con una densidad de muestreo promedio de $4 \mathrm{~km}^{2}$ y fueron analizadas por Espectrometría de Masas con Plasma Acoplado Inductivamente (ICP-MS) y Fluorescencia De Rayos X (XRF) por 39 elementos incluyendo óxidos mayores; datos que están disponibles y de libre descarga en el portal web del INGEMMET.

\subsection{Metodología}

Los datos de las 3146 muestras fueron sometidos a un análisis exploratorio, en el cual se identificó que la mayoría de los elementos presentan distribución log-normal, se identificó también, el porcentaje de datos censurados (valores por debajo del límite de detección) un total de 1.4\% de datos censurados; los elementos con mayor porcentaje de datos censurados fueron: $\mathrm{Cd}(13 \%), \mathrm{Bi}(12 \%)$, Mo $(11 \%), \mathrm{Nb}(11 \%), \mathrm{Au}(4 \%), \mathrm{U}(4 \%), \mathrm{Cr}(1 \%)$ y $\mathrm{Sb}(1 \%)$. Considerando que ningún elemento supera el $15 \%$ de datos censurados se realizó una imputación de valores, para cada dato censurado, usando el método de regresión de orden estadística robusta (rROS, por sus siglas en inglés) que asume una distribución log-normal (Helsel, 2011; Quintanilla Casas, 2017) en el software libre ProUCL 5.1.

Con los datos uniformizados se elaboró mapas de anomalías usando la metodología tradicional, para lo cual las 3146 muestras se separaron por dominios geológicos y se determinó, para cada caso, los parámetros geoquímicos como el valor de fondo, umbrales y anomalías basados en las estadísticas descriptivas, media y desviación estándar, de los datos normalizados mediante logaritmo natural. Esto nos servirá como punto de comparación con la nueva metodología propuesta en este trabajo.

Los parámetros geoquímicos se determinaron de acuerdo con:

- $\quad$ Valor de fondo = Media aritmética

- Umbral 1 = Media +1 desviación estándar

- $\quad$ Umbral 2 = Media +2 desviaciones estándar

- $\quad$ Umbral 3 = Media +3 desviaciones estándar

- Anomalía débil = valores entre Umbral $1 \mathrm{y}$ Umbral 2 


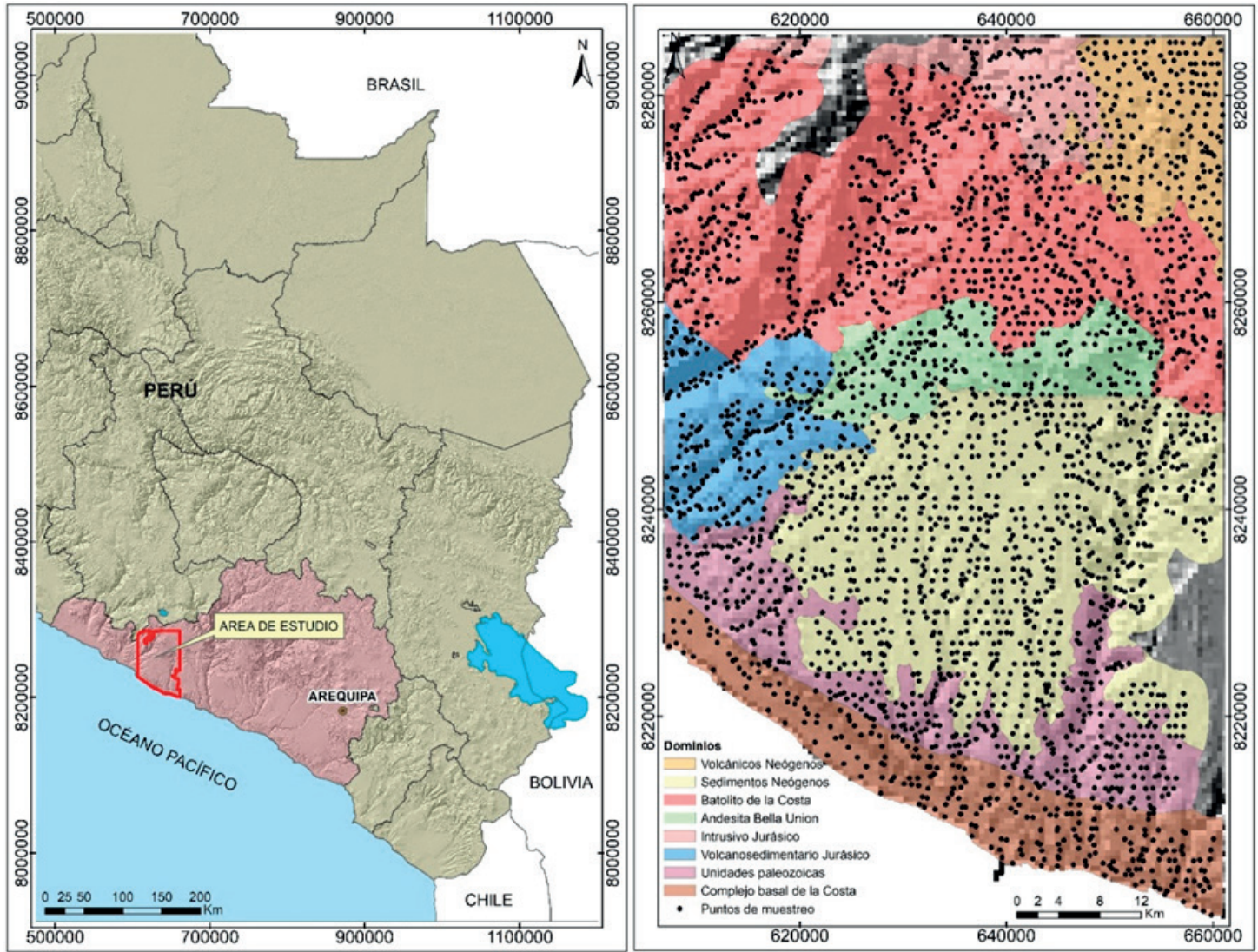

Figura 1. Izquierda: Mapa de ubicación del área de estudio. Derecha: Mapa geológico simplificado por dominios geológicos mostrando los puntos de muestreo.

- $\quad$ Anomalía moderada $=$ valores entre Umbral 2 y Umbral 3

\section{- $\quad$ Anomalía fuerte $=$ valores mayores a Umbral 3}

Dados que los valores están afectados por logaritmo natural para obtener el valor en ppm o ppb, según corresponda, se usó la función exponencial.

Los valores de $\mathrm{Cu}$ y $\mathrm{Au}$ se interpolaron por dominio geológico usando el software ArcMap 10.5 mediante distancia inversa ponderada (IDW) y se clasificaron de acuerdo con los valores de los parámetros geoquímicos obtenidos en cada dominio.

Luego se calculó el LREI para elementos indicadores de mineralización: $\mathrm{Cu}, \mathrm{Au}, \mathrm{Ag}, \mathrm{Pb}, \mathrm{Zn}, \mathrm{Mo}$ usando una ventana deslizante de $10 \mathrm{~km}$ x $10 \mathrm{~km}$.

$$
\operatorname{LREI}(X)=\frac{X_{i}}{\text { Mediana }_{j}}-1
$$

$X_{i}$ representa la concentración del elemento en la

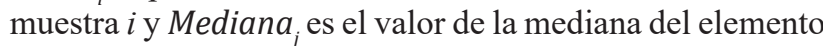
$X$ dentro de la ventana $j$.

El método de la ventana deslizante permite controlar la variabilidad espacial de los datos y la variación del valor de fondo dentro de un vecindario local (Figura 2).
Para el análisis multivariado se usó la transformación log-ratio centrada (CLR) con la finalidad de abrir y normalizar los datos (Aitchison \& Jones, 1987)

$$
\operatorname{clr}(X)=\left(\log \frac{X_{1}}{g(X)}, \log \frac{X_{2}}{g(X)}, \ldots \log \frac{X_{i}}{g(X)}\right)
$$

$X_{i}$ representa la concentración del elemento en la muestra $i$ y $\mathrm{g}(\mathrm{X})$ es la media geométrica.

Luego se determinó los coeficientes de rango correlacional (RCC, por sus siglas en inglés) siguiendo la metodología propuesta por Valls (2008) y el análisis de componentes principales (PCA) usando el software IoGAS 7.3. Se consideró ambos análisis multivariados debido a que el RCC se basa en los coeficientes de correlación de Pearson y el PCA se basa en la varianza de los datos.

Finalmente se interpoló los LREI, RCC y PCA usando el software ArcMap 10.5 mediante distancia inversa ponderada (IDW) y se generaron rasters con pixeles de $100 \mathrm{~m} \times 100 \mathrm{~m}$ los cuales se reclasificaron a valores entre 0 y 1 a través de la clasificación de pertenencia FuzzyLarge para realizar la superposición difusa (Figura 3).

Con los rasters de entrada usados para la superposición difusa se muestreó cada píxel y los datos se emplearon para generar un mapa auto organizado (SOM por sus siglas en inglés), un tipo de red neuronal artificial que es entrenada 


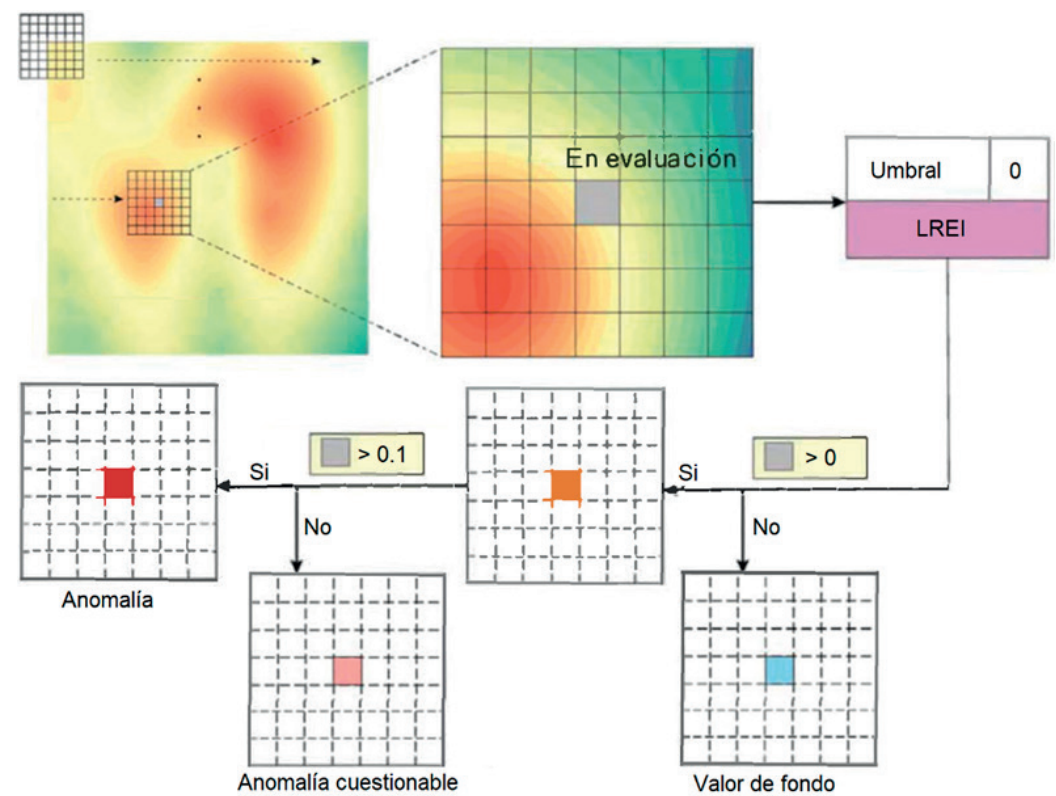

Figura 2. Esquema que muestra el flujo de evaluación del LREI por el método de ventana deslizante. Modificado de Wang \& Zuo (2016)

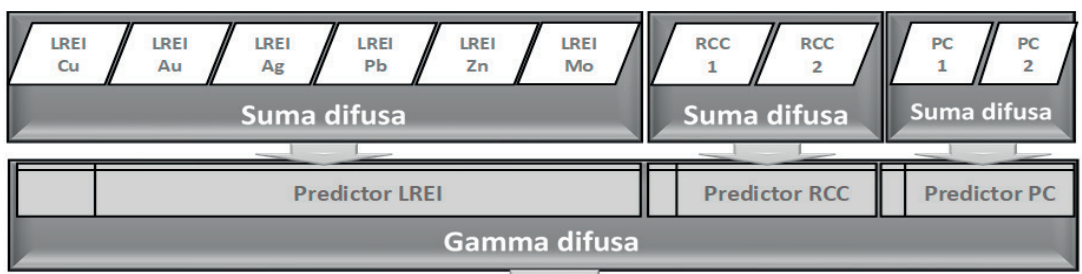

Mapa de prospectividad geoquímica

Figura 3. Diagrama de flujo para generar el mapa de prospectividad geoquímica a través de superposiciones difusas (Fuzzy modelling)

usando aprendizaje no supervisado, usando el software IoGAS 7.3.

El mapa de prospectividad geoquímica de la superposición difusa y el mapa autoorganizado fueron validados con las ocurrencias y depósitos minerales conocidos.

\section{RESULTADOS}

\subsection{Parámetros geoquímicos - metodología tradicional}

Los parámetros geoquímicos de $\mathrm{Cu}$ y Au obtenidos mediante la metodología tradicional se muestran en la tabla 1.

Los mapas geoquímicos de $\mathrm{Cu}$ y $\mathrm{Au}$ se muestran en la Figura 4, donde se puede observar que varios depósitos minerales conocidos se plotean dentro de áreas con anomalías débiles, moderadas o fuertes, además los mapas presentan áreas con moderadas y fuertes anomalías en las que no hay depósitos minerales conocidos, las cuales se pueden considerar como posibles targets exploratorios; sin embargo hay algunos depósitos minerales que se plotean dentro de áreas con valores de fondo o debajo del valor de fondo, esto es debido a que la metodología tradicional sólo permite identificar anomalías geoquímicas que son evidentes, dado que no considera la variabilidad espacial de los datos y la variación del valor de fondo dentro de los dominios geológicos, oculta aquellas anomalías débiles o muy débiles que no son tan evidentes.

\section{2. Índice de enriquecimiento relativo local - LREI}

La cartografía geoquímica de los índices de enriquecimiento relativo local (LREI) de $\mathrm{Cu}, \mathrm{Au}, \mathrm{Ag}, \mathrm{Pb}, \mathrm{Zn}$ y $\mathrm{Mo}$ en los cuadrángulos de Chaparra y Atico (Figura 5) muestra zonas anómalas que demarcan la localización de los depósitos minerales conocidos (símbolos en negro en la figura 5) concernientes a vetas auríferas, pórfidos de $\mathrm{Cu}$ Mo, Epitermales de $\mathrm{Au}-\mathrm{Ag}$ y depósitos de $\mathrm{Au}(\mathrm{Pb}, \mathrm{Zn}, \mathrm{Cu})$ relacionados a intrusiones, y además se hacen evidentes zonas anómalas que en la cartografía de los parámetros geoquímicos del método tradicional (Figura 4) estaban ocultas. Estas nuevas zonas pueden estar asociadas a ocurrencias minerales de interés exploratorio.

\subsection{Coeficientes de rango correlacional- RCC}

Usando los coeficientes de correlación significativos (con T-student > 3, como sugiere Valls, (2008), se determinó dos coeficientes de rango correlacional más importantes: 
Tabla 1. Parámetros geoquímicos de Cu y Au por dominios geológicos

\begin{tabular}{|c|c|c|c|c|c|c|c|c|c|c|c|c|c|}
\hline \multirow{2}{*}{$\begin{array}{l}\text { Dominios } \\
\text { geológicos }\end{array}$} & \multirow{2}{*}{$\begin{array}{c}\mathrm{N}^{\circ} \text { de } \\
\text { muestras }\end{array}$} & \multicolumn{2}{|c|}{$\operatorname{Ln}(\mathrm{Cu})$} & \multicolumn{4}{|c|}{ Cu_ppm } & \multicolumn{2}{|c|}{$\operatorname{Ln}(\mathrm{Au})$} & \multicolumn{4}{|c|}{ Au_ppb } \\
\hline & & Media & $\begin{array}{l}\text { Desv. } \\
\text { Estándar }\end{array}$ & $\begin{array}{l}\text { Valor de } \\
\text { fondo }\end{array}$ & $\underset{1}{\text { Umbral }}$ & $\underset{2}{\text { Umbral }}$ & $\underset{3}{\text { Umbral }}$ & Media & $\begin{array}{l}\text { Desv. } \\
\text { Estándar }\end{array}$ & $\begin{array}{l}\text { Valor de } \\
\text { fondo }\end{array}$ & $\underset{1}{\text { Umbral }}$ & $\underset{2}{\text { Umbral }}$ & $\underset{3}{\text { Umbral }}$ \\
\hline $\begin{array}{l}\text { Complejo Basal de } \\
\text { la Costa }\end{array}$ & 284 & 3.20 & 0.46 & 24.45 & 38.74 & 61.38 & 97.26 & -0.18 & 1.01 & 0.83 & 2.29 & 6.28 & 17.24 \\
\hline $\begin{array}{l}\text { Unidades } \\
\text { Paleozoicas }\end{array}$ & 389 & 3.73 & 0.69 & 41.62 & 83.23 & 166.43 & 332.82 & -0.09 & 0.96 & 0.91 & 2.39 & 6.24 & 16.32 \\
\hline $\begin{array}{l}\text { Volcanosedimentario } \\
\text { Jurásico }\end{array}$ & 216 & 4.38 & 0.58 & 80.19 & 142.68 & 253.85 & 451.65 & 0.02 & 0.84 & 1.02 & 2.36 & 5.49 & 12.75 \\
\hline Intrusivo Jurásico & 107 & 3.98 & 0.62 & 53.44 & 99.70 & 186.01 & 347.04 & 0.97 & 1.18 & 2.65 & 8.63 & 28.10 & 91.53 \\
\hline Andesita Bella Union & 230 & 4.03 & 0.77 & 56.50 & 121.45 & 261.06 & 561.15 & -0.10 & 1.06 & 0.90 & 2.61 & 7.52 & 21.70 \\
\hline Batolito de la Costa & 865 & 3.19 & 0.58 & 24.33 & 43.25 & 76.88 & 136.66 & 0.40 & 1.26 & 1.49 & 5.27 & 18.60 & 65.68 \\
\hline $\begin{array}{l}\text { Sedimentos } \\
\text { neógenos }\end{array}$ & 844 & 3.65 & 0.68 & 38.48 & 75.98 & 150.02 & 296.23 & 0.35 & 0.93 & 1.41 & 3.60 & 9.15 & 23.26 \\
\hline Volcánico neógeno & 211 & 3.30 & 0.65 & 27.16 & 51.91 & 99.22 & 189.66 & 0.10 & 1.08 & 1.10 & 3.26 & 9.66 & 28.57 \\
\hline Total de muestras & 3146 & 3.56 & 0.73 & 35.17 & 72.73 & 150.40 & 311.02 & 0.21 & 1.10 & 1.23 & 3.68 & 11.02 & 32.97 \\
\hline
\end{tabular}
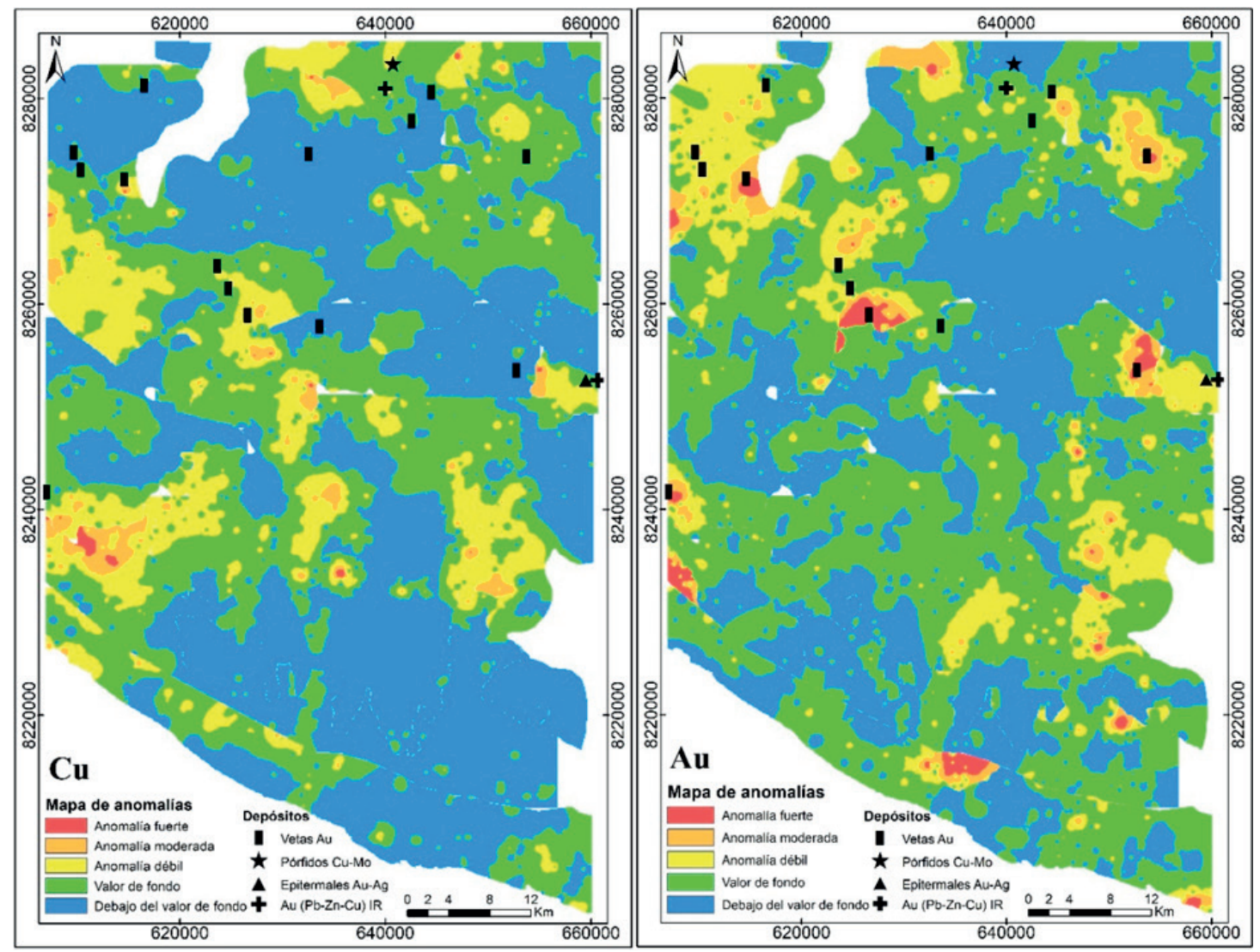

Figura 4. Izquierda: Mapa de anomalías de Cu. Derecha: Mapa de anomalías de Au. Usando la metodología tradicional

El RCC1 asocia elementos calcófilos con siderófilos inversamente correlacionados con elementos litófilos, por la estructura y distribución espacial este coeficiente representa posibles zonas mineralizadas cupríferas que pueden estar asociados a depósitos porfiríticos o IOCG (Figura 6).

El RCC2 asocia a elementos pathfinder (As-Cd) que pueden representar posibles zonas auríferas y/o polimetálicos relacionados a sistemas magmáticohidrotermales (Figura 6).

\subsection{Análisis de componentes principales - PCA}

Con el análisis de componentes principales (PCA) se obtuvo 10 componentes principales con valor propio mayor a la unidad que explican el $76.8 \%$ de la varianza total de los datos, de las cuales PC1 y PC2 (Figuras 7 y 9) explican el $21 \%$ y $15.7 \%$ de la varianza respectivamente y asocian, el PC1 elementos relacionados a mineralización cuprífera $(\mathrm{Cu}, \mathrm{V}, \mathrm{Mg}, \mathrm{Fe}, \mathrm{Co}, \mathrm{Zn})$ y PC2 elementos relacionados a mineralización de Au y Ag ( $\mathrm{Au}, \mathrm{Ag}, \mathrm{Bi}, \mathrm{Sb}, \mathrm{Ca}, \mathrm{Hg}, \mathrm{W}, \mathrm{As}$ ) (Figuras 8 y 9). 


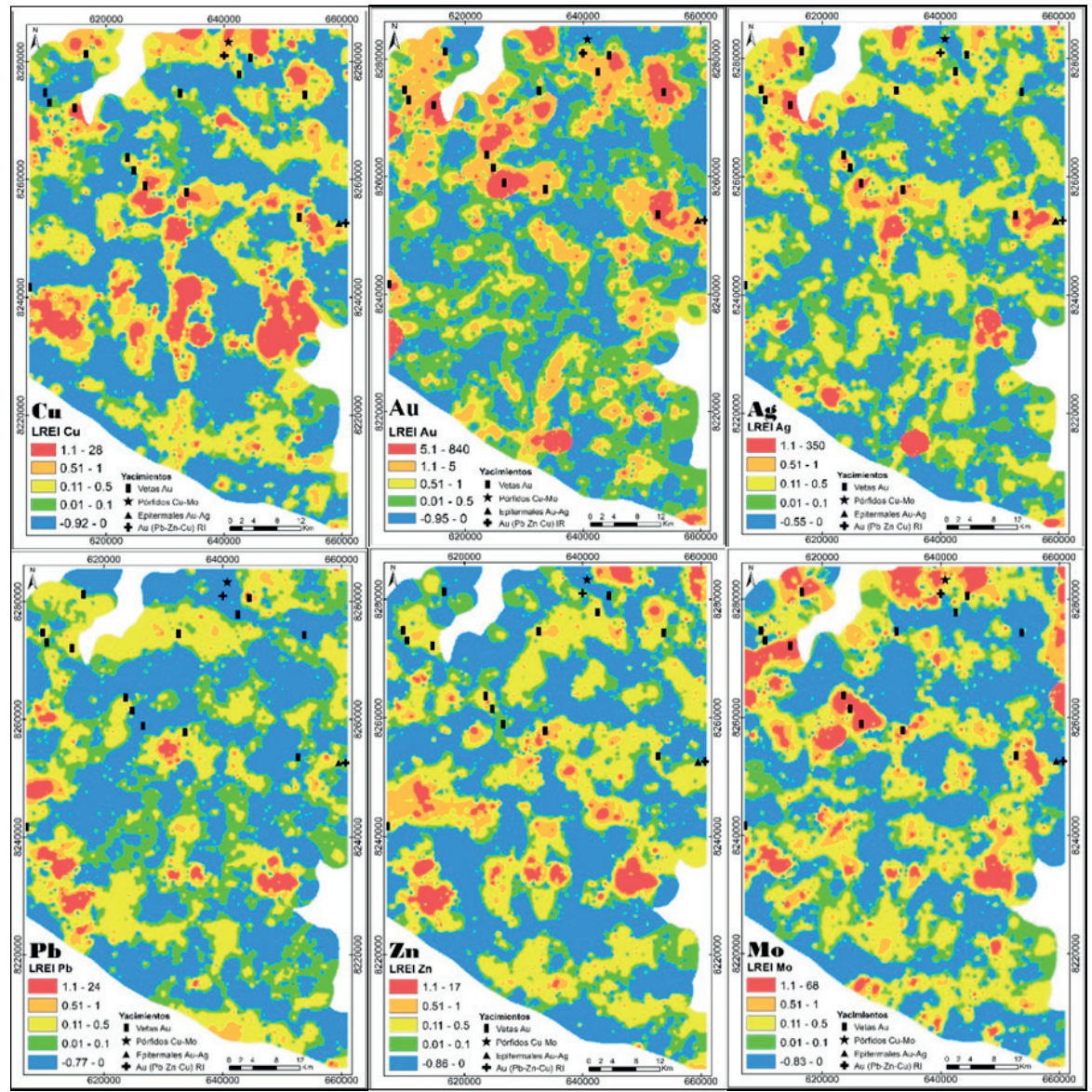

Figura 5. Mapas de índice de enriquecimiento relativo local (LREI) de $\mathrm{Cu}, \mathrm{Au}, \mathrm{Ag}, \mathrm{Pb}, \mathrm{Zn}$ y Mo.
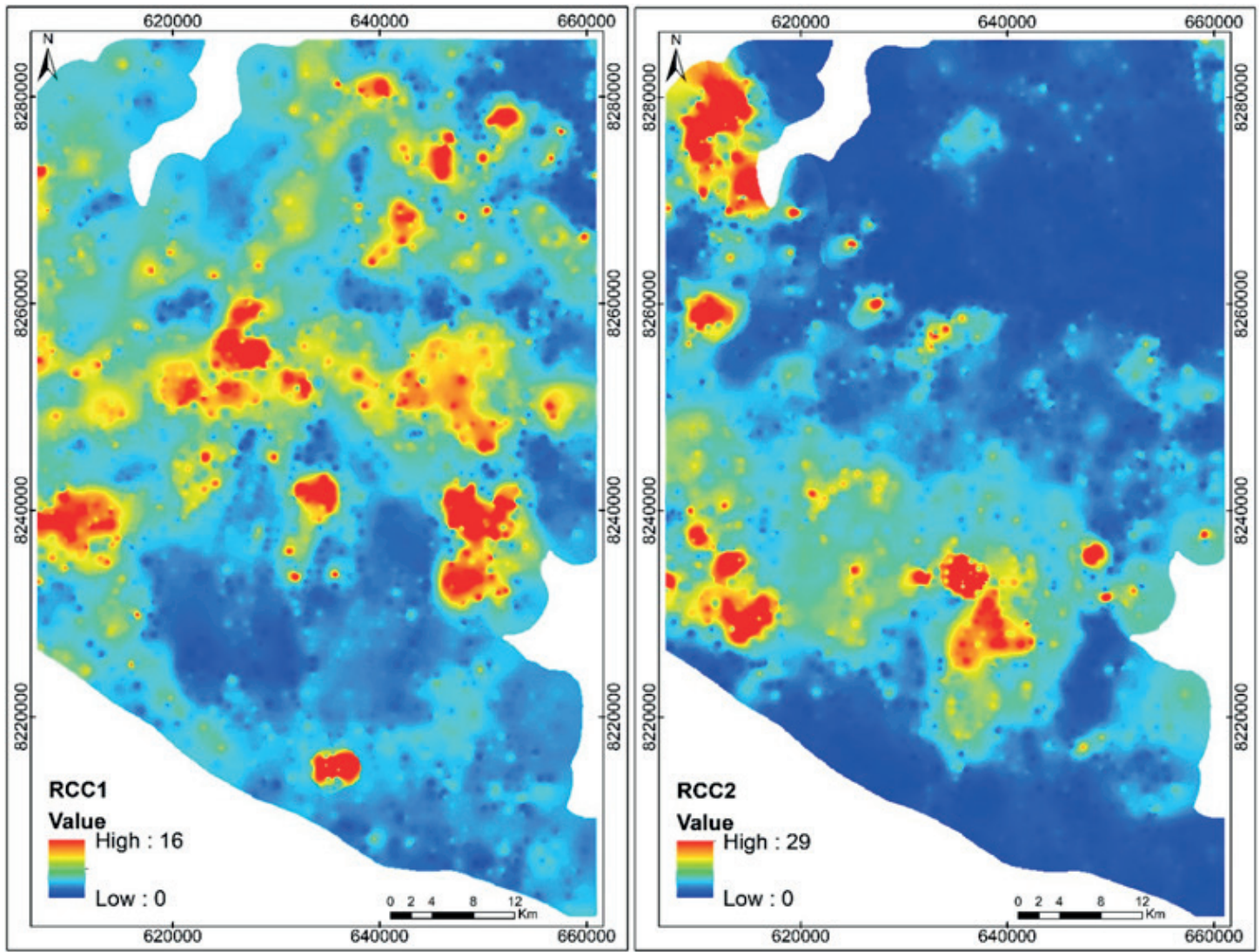

Figura 6. Mapas de distribución de los coeficientes de rango correlacional RCC1 Y RCC2 (ver texto) 

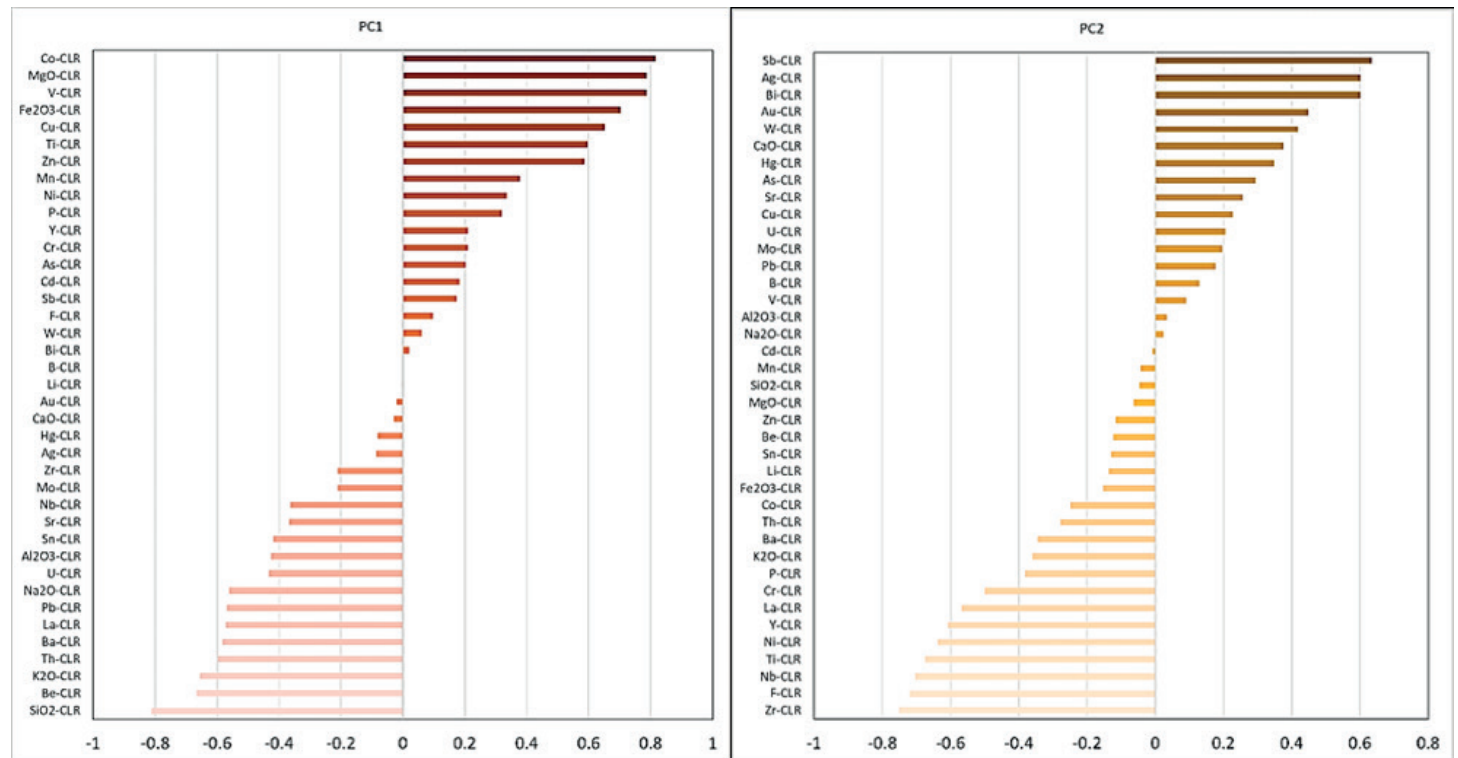

Figura 7. Diagrama de barras de las puntuaciones escaladas de los componentes principales PC1 (izquierda) y PC2 (derecha)

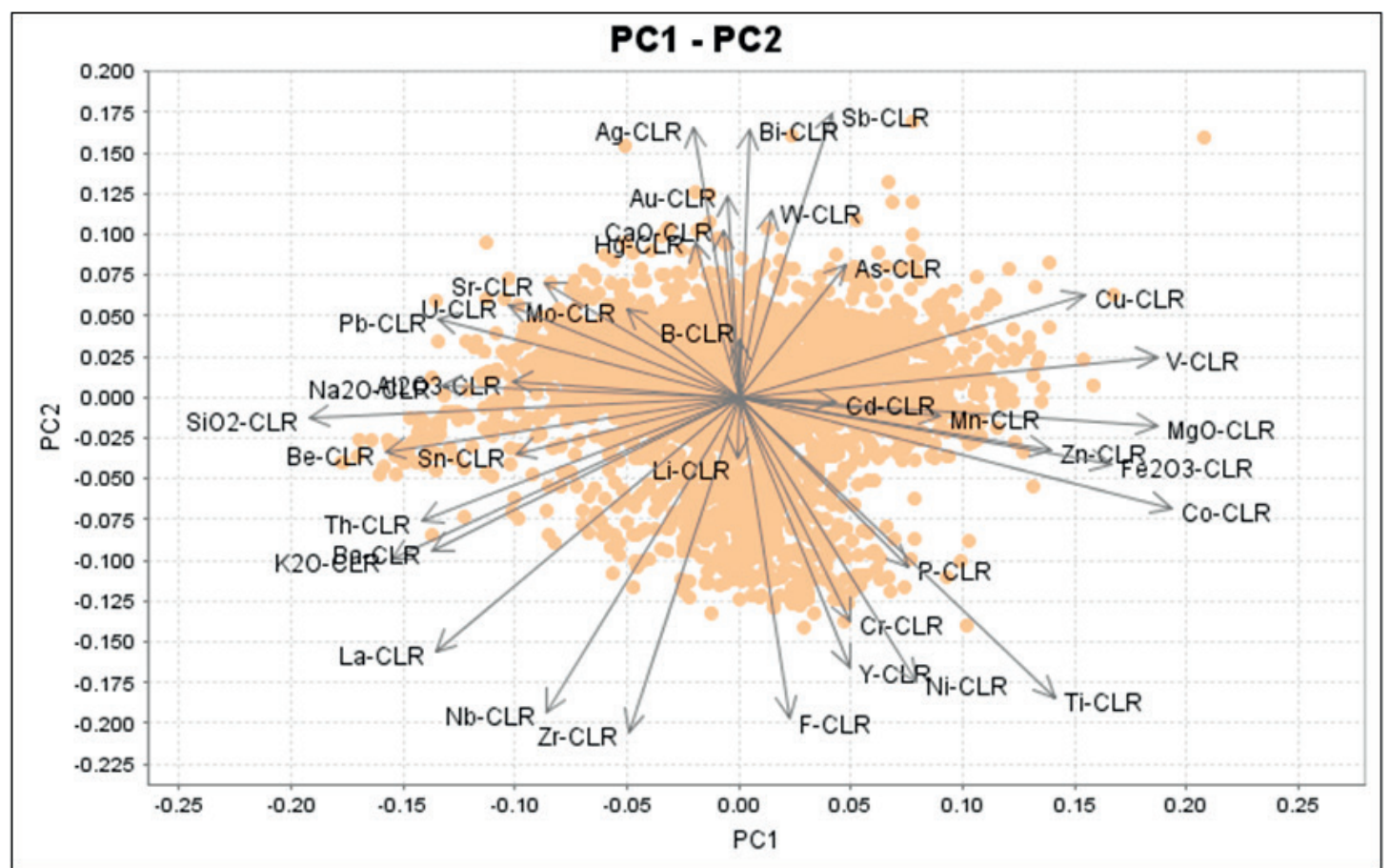

Figura 8. Gráfica de cargas de las componentes principales PC1 y PC2 en los que se observan los principales elementos asociados en cada PC.

\subsection{Mapas de prospectividad geoquímica}

La superposición Suma difusa de los rasters generados mediante la interpolación IDW de cada uno de los LREI, RCC y PC y reclasificados mediante la clasificación de pertenencia FuzzyLarge permitió obtener tres mapas predictores, predictor LREI, predictor RCC y predictor $\mathrm{PC}$ los que fueron superpuestos mediante superposición Gamma ( $\gamma=90)$, obteniendo el mapa de prospectividad Fuzzy (Figura 10, izquierda), el nivel de prospectividad se determinó estadísticamente, considerando el valor de fondo igual a la media y un umbral de la media más una desviación estándar, siendo así, un nivel bajo aquellos valores menores a la media, nivel medio aquellos valores entre la media y el umbral, y un nivel alto de prospectividad aquellos valores mayores al umbral.

Aplicando las redes neuronales artificiales se generó un mapa autoorganizado (SOM) tomando como datos de entrada los valores de los pixeles de cada mapa de los LREI, RCC y PC, haciendo un total de 384421 pixeles muestreados; la inteligencia artificial, aprendizaje automático y aprendizaje profundo permiten procesar una gran cantidad de datos y producir un mapa. Los valores de 


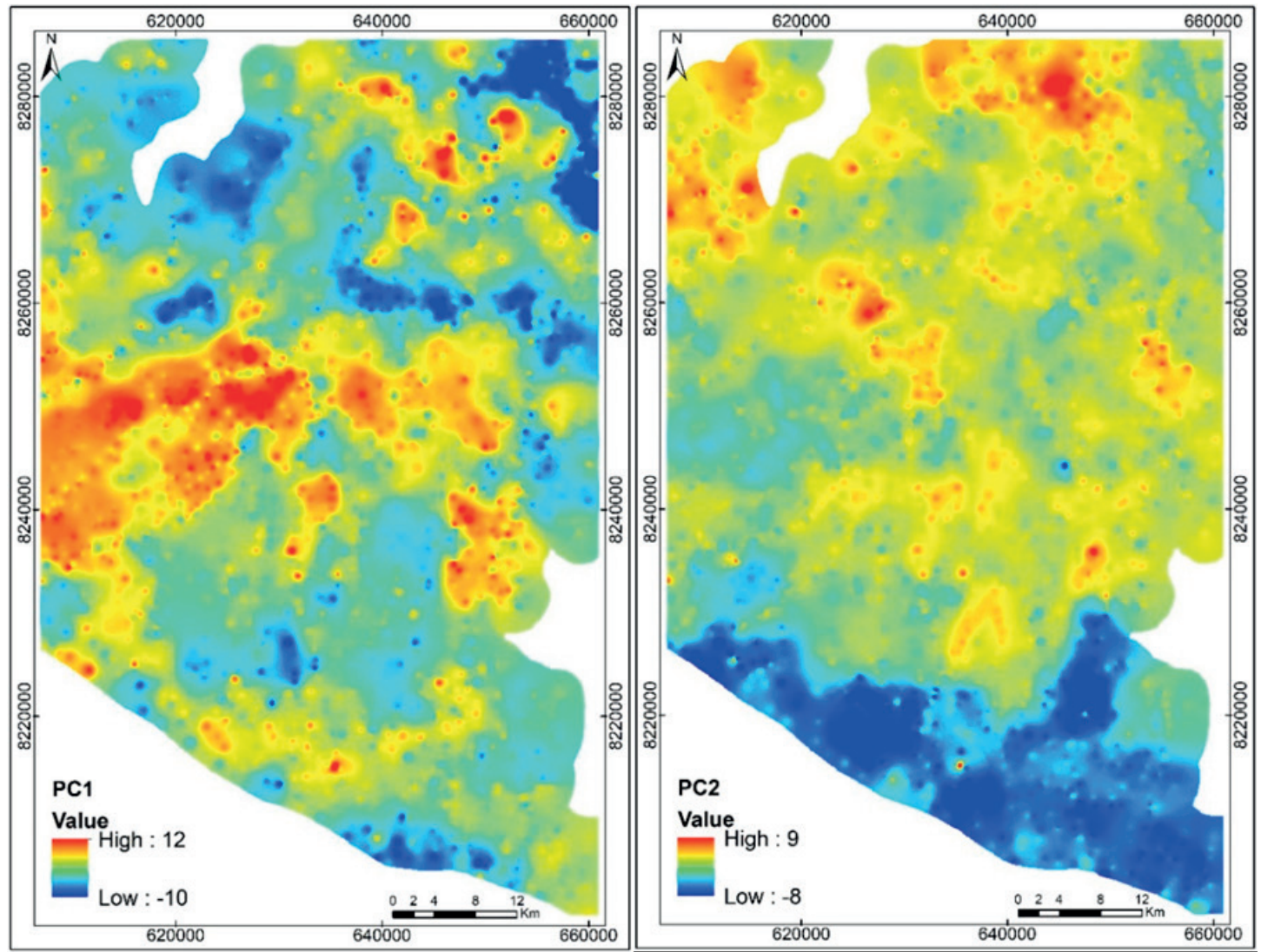

Figura 9. Mapa de distribución de las puntuaciones de las componentes principales PC1 y PC2.

calidad SOM generados en IoGAS 7.3 fueron interpolados mediante IDW en ArcMap para producir un mapa de prospectividad SOM (Figura 10, derecha), cuyo nivel de prospectividad se determinó estadísticamente, esta vez, dado que los datos presentan distribución log-normal, se consideró el valor de fondo igual a la mediana y el umbral igual al tercer cuartil más 1.5 el rango intercuartil (Q3 + 1.5 RIQ). Siendo entonces, un nivel bajo aquellos valores menores a la mediana, nivel medio aquellos valores entre la mediana y el umbral, y un nivel alto de prospectividad aquellos valores mayores al umbral.

Los mapas de prospestividad Fuzzy y SOM fueron validados ploteando las coordenadas de 109 depósitos metálicos conocidos incluyendo las ocurrencias minerales, los resultados muestran que las áreas con alta o moderada prospectividad Fuzzy que cubren un 38\% del área de estudio contienen $75 \%$ de los depósitos conocidos y las áreas con alta o moderada prospectividad SOM cubren un 50\% del área de estudio y contienen $72 \%$ de los depósitos conocidos (Tabla 2), lo que valida el modelo y además permite sugerir nuevas zonas con alto nivel de prospectividad y que pueden ser de interés exploratorio.

En color rojo se muestra las áreas con alto nivel de prospectividad, en color amarillo las áreas con moderado nivel de prospectividad y color azul las áreas con bajo nivel de prospectividad. Los símbolos en negro corresponden a las coordenadas de los depósitos minerales conocidos dentro del área.
Tabla 2. Validación de los mapas de prospectividad Fuzzy y SOM con los depósitos conocidos

\begin{tabular}{lcccc}
\hline Prospectividad & \multicolumn{2}{c}{ Fuzzy } & \multicolumn{2}{c}{ SOM } \\
& $\begin{array}{c}\text { Área potencial } \\
(\%)\end{array}$ & $\begin{array}{c}\text { Ocurrencias } \\
(\%)\end{array}$ & $\begin{array}{c}\text { Área potencial } \\
(\%)\end{array}$ & $\begin{array}{c}\text { Ocurrencias } \\
(\%)\end{array}$ \\
\hline Alto & $15 \%$ & $36 \%$ & $12 \%$ & $29 \%$ \\
Moderado & $23 \%$ & $39 \%$ & $39 \%$ & $43 \%$ \\
Bajo & $62 \%$ & $25 \%$ & $50 \%$ & $28 \%$ \\
\hline
\end{tabular}

\section{DISCUSIÓN}

La metodología tradicional de prospección geoquímica usando los parámetros estadísticos, valor de fondo y umbral, propuesto por HAWKES \& WEBB (1963), permitieron identificar las anomalías geoquímicas más evidentes, sin considerar la variabilidad espacial de los datos ni la variación del valor de fondo dentro de los dominios geológicos, sin embargo ocultan las anomalías débiles o muy débiles, las mismas que se hacen evidentes en la cartografía de los índices de enriquecimiento relativo local (LREI), tal como lo propone Zuo (2014).

El índice de enriquecimiento relativo local (LREI) de los elementos indicadores $\mathrm{Cu}, \mathrm{Au}, \mathrm{Ag}, \mathrm{Pb}, \mathrm{Zn}$ y Mo usando una ventana deslizante de $10 \mathrm{~km}$ x $10 \mathrm{~km}$ puso en evidencia nuevas áreas anómalas que en el método tradicional no se observaba. 

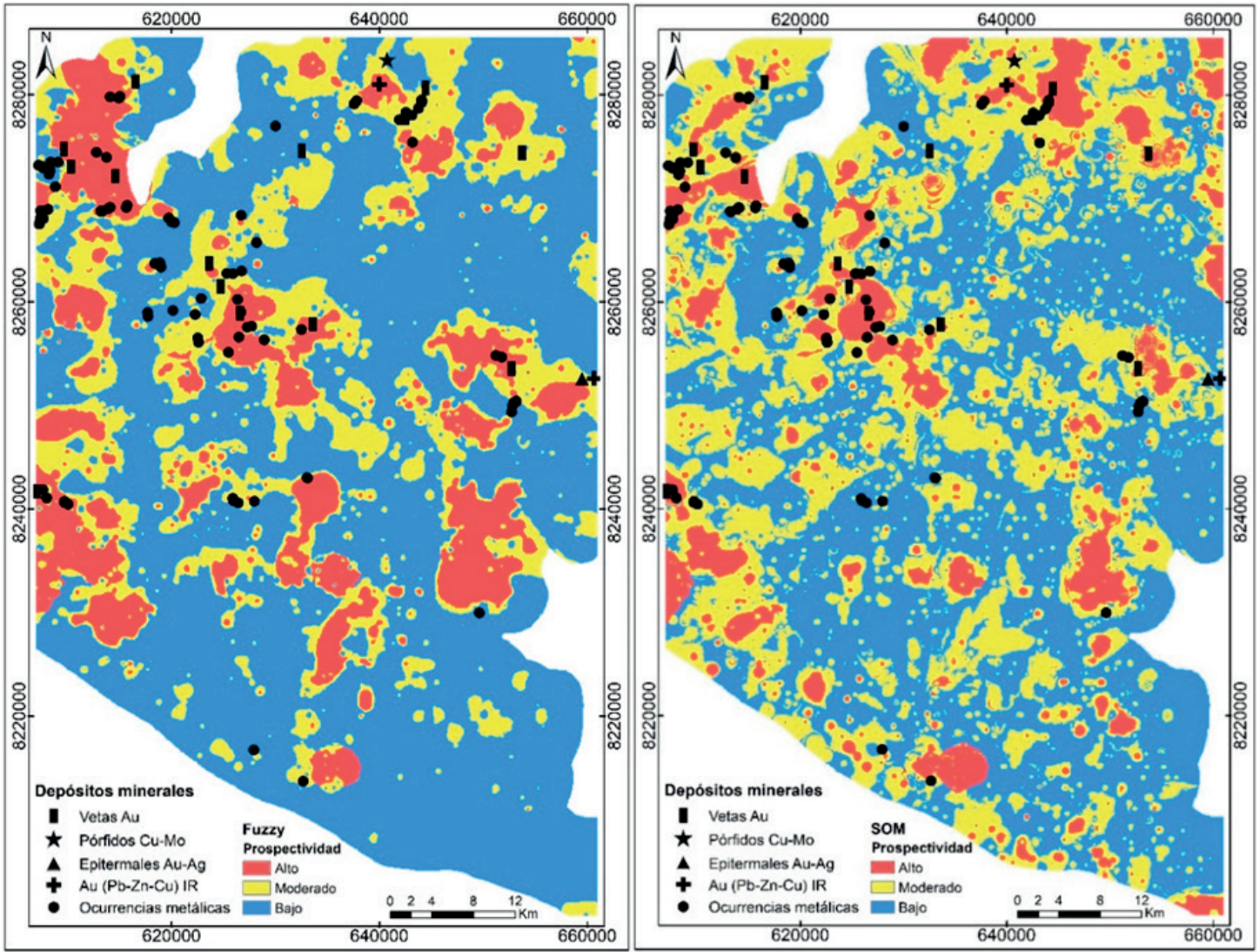

Figura 10. Izquierda: Mapa de prospectividad Fuzzy. Derecha: Mapa de prospectividad SOM.

Los coeficientes de rango correlacional propuesto por Valls (2008), y el análisis de componentes principales usado en varias partes del mundo para exploración geoquímica (Zuo, 2011; Cheng et al., 2011; Zuo et al., 2013; Chen et al., 2019) ayudan a determinar asociaciones geoquímicas que permiten sugerir que el área en estudio es prospectiva por depósitos cupríferos como pórfidos y/o IOCG y depósitos de metales base $\mathrm{Au} \mathrm{y} \mathrm{Ag} \mathrm{relacionados} \mathrm{a} \mathrm{sistemas}$ magmático-hidrotermales, ya sea epitermales u otros.

Los mapas de prospectividad geoquímica aplicando técnicas modernas como Fuzzy y SOM, que integran la información estadística univariable y multivariable de los datos geoquímicos, muestran zonas con moderada y alta prospectividad que fueron validados con los depósitos minerales conocidos, lo cual permite sugerir nuevas áreas de interés exploratorio en los cuadrángulos de Chaparra y Atico. Asimismo, estos mapas pueden ser integrados junto con mapas de litología, estructural, teledetección y geofísica usando herramientas similares para generar mapas de prospectividad geológica de mayor precisión como se observa en Yousefi et al., 2014; Moradi et al., 2015; Zuo et al., 2019.

\section{CONCLUSIONES}

Las innovaciones estadísticas univariables y multivariables como el índice de enriquecimiento relativo local (LREI), el coeficiente de rango correlacional (RCC), el análisis de componentes principales (PCA), y las innovaciones tecnológicas aplicadas a la cartografía geoquímica como la superposición difusa (Fuzzy) y los mapas autoorganizados (SOM) que usa las redes neuronales artificiales, son herramientas efectivas para la prospección geoquímica.

Los mapas de prospectividad tanto Fuzzy como SOM generados a partir de las muestras de sedimentos de corriente en los cuadrángulos de Chaparra y Atico, Caravelí - Arequipa evidencian la efectividad de la metodología aplicada, ya que demarcan con alta precisión la ubicación de los depósitos y ocurrencias minerales conocidas y además muestran otras áreas con alto nivel de prospectividad que pueden ser de interés exploratorio.

\section{AGRADECIMIENTOS}

Un agradecimiento especial a los ingenieros Alberto Torres y Víctor Torres por sus comentarios y sugerencias, que ayudaron en gran manera a mejorar este documento. Se hace extensivo el agradecimiento a IMDEX por facilitarnos la licencia del software IoGAS 7.3.

\section{REFERENCIAS}

Aitchison, J., \& Jones, M. C. (1987). The Statistical Analysis of Compositional Data. Journal of the Royal Statistical Society. Series A (General), 150(4), 396. https://doi. org $/ 10.2307 / 2982045$ 
Chen, S., Plouffe, A., \& Hattori, K. (2019). A multivariate statistical approach identifying the areas underlain by potential porphyry-style $\mathrm{Cu}$ mineralization, south-central British Columbia, Canada. Journal of Geochemical Exploration, 202, 13-26. https://doi.org/10.1016/j.gexplo.2019.03.016

Cheng, Q. (2007). Mapping singularities with stream sediment geochemical data for prediction of undiscovered mineral deposits in Gejiu, Yunnan Province, China. Ore Geology Reviews, 32(1-2), 314-324. https://doi.org/10.1016/j. oregeorev.2006.10.002

Cheng, Q., Bonham-Carter, G., Wang, W., Zhang, S., Li, W., \& Qinglin, X. (2011). A spatially weighted principal component analysis for multi-element geochemical data for mapping locations of felsic intrusions in the Gejiu mineral district of Yunnan, China. Computers and Geosciences, 37(5), 662669. https://doi.org/10.1016/j.cageo.2010.11.001

HAWKES, H. E., \& WEBB, J. S. (1963). Geochemistry in Mineral Exploration. Soil Science, 95(4), 283. https://doi. org/10.1097/00010694-196304000-00016

Helsel, D. R. (2011). Statistics for Censored Environmental Data Using Minitab ${ }^{\circledR}$ and R: Second Edition. In Statistics for Censored Environmental Data Using Minitab ${ }^{\circledR}$ and R: Second Edition (Vol. 14, Issue 1). https://doi. org/10.1002/9781118162729

Kirkwood, C., Cave, M., Beamish, D., Grebby, S., \& Ferreira, A. (2016). A machine learning approach to geochemical mapping. Journal of Geochemical Exploration, 167, 49-61. https://doi.org/10.1016/j.gexplo.2016.05.003

Liu, Y., Cheng, Q., Zhou, K., Xia, Q., \& Wang, X. (2016). Multivariate analysis for geochemical process identification using stream sediment geochemical data: A perspective from compositional data. Geochemical Journal, 50(4), 293-314. https://doi.org/10.2343/geochemj.2.0415

Moradi, M., Basiri, S., Kananian, A., \& Kabiri, K. (2015). Fuzzy logic modeling for hydrothermal gold mineralization mapping using geochemical, geological, ASTER imageries and other geo-data, a case study in Central Alborz, Iran. Earth Science Informatics, 8(1), 197-205. https://doi. org/10.1007/s12145-014-0151-9

Quintanilla Casas, B. (2017). Estadística en variables con censura: aplicación a datos medioambientales. http:// openaccess.uoc.edu/webapps/o2/handle/10609/63786

Reimann, C., Filzmoser, P., \& Garrett, R. G. (2002). Factor analysis applied to regional geochemical data: Problems and possibilities. Applied Geochemistry, 17(3), 185-206. https:// doi.org/10.1016/S0883-2927(01)00066-X

Torres, D., Santos, A., Ccallo, W., \& Soberón, O. (2017). Geología de los cuadrángulos de Atico y Cháparra Hojas 330 y 320. INGEMMET, Boletin Serie A: Carta Geológica Nacional $\mathrm{N}^{\circ}$ 149. Repositorio Institucional INGEMMET. https:// repositorio.ingemmet.gob.pe/handle/20.500.12544/1009

Tripathi, V. S. (1979). Factor analysis in geochemical exploration. Journal of Geochemical Exploration, 11(3), 263-275. https://doi.org/10.1016/0375-6742(79)90004-9

Valls, R. A. (2008). Why, and how, we should use compositional data analysis. A step-by-step guide for the field geologists. https://www.researchgate.net/publication/269104323 WHY_AND_HOW_WE_SHOULD_USE
COMPOSITIONAL DATA ANALYSIS A Step-byStep_Guide_for_the_Field_Geologists_Special_Edition_ for_Wikibooks_January_2_nd_2008

Wang, J., \& Zuo, R. (2016). An extended local gap statistic for identifying geochemical anomalies. Journal of Geochemical Exploration, 164, 86-93. https://doi.org/10.1016/j. gexplo.2016.01.002

Yousefi, M., Kamkar-Rouhani, A., \& Carranza, E. J. M. (2014). Application of staged factor analysis and logistic function to create a fuzzy stream sediment geochemical evidence layer for mineral prospectivity mapping. Geochemistry: Exploration, Environment, Analysis, 14(1), 45-58. https:/ doi.org/10.1144/geochem2012-144

Zhang, C., Jordan, C., \& Higgins, A. (2007). Using neighbourhood statistics and GIS to quantify and visualize spatial variation in geochemical variables: An example using Ni concentrations in the topsoils of Northern Ireland. Geoderma, 137(3-4), 466-476. https://doi.org/10.1016/j.geoderma.2006.10.018

Ziaii, M., Pouyan, A. A., \& Ziaei, M. (2009). Neuro-fuzzy modelling in mining geochemistry: Identification of geochemical anomalies. Journal of Geochemical Exploration, 100(1), 25-36. https://doi.org/10.1016/j. gexplo.2008.03.004

Zuo, R. (2011). Identifying geochemical anomalies associated with $\mathrm{Cu}$ and $\mathrm{Pb}-\mathrm{Zn}$ skarn mineralization using principal component analysis and spectrum-area fractal modeling in the Gangdese Belt, Tibet (China). Journal of Geochemical Exploration, 111(1-2), 13-22. https://doi.org/10.1016/j. gexplo.2011.06.012

Zuo, R. (2014). Identification of weak geochemical anomalies using robust neighborhood statistics coupled with GIS in covered areas. Journal of Geochemical Exploration, 136, 93-101. https://doi.org/10.1016/j.gexplo.2013.10.011

Zuo, R. (2017). Machine Learning of Mineralization-Related Geochemical Anomalies: A Review of Potential Methods. In Natural Resources Research (Vol. 26, Issue 4, pp. 457-464). https://doi.org/10.1007/s11053-017-9345-4

Zuo, R., \& Wang, J. (2016). Fractal/multifractal modeling of geochemical data: A review. In Journal of Geochemical Exploration (Vol. 164, pp. 33-41). https://doi.org/10.1016/j. gexplo.2015.04.010

Zuo, R., Xia, Q., \& Wang, H. (2013). Compositional data analysis in the study of integrated geochemical anomalies associated with mineralization. Applied Geochemistry, 28, 202-211. https://doi.org/10.1016/j.apgeochem.2012.10.031

Zuo, R., \& Xiong, Y. (2018). Big Data Analytics of Identifying Geochemical Anomalies Supported by Machine Learning Methods. Natural Resources Research, 27(1), 5-13. https:// doi.org/10.1007/s11053-017-9357-0

Zuo, R., Xiong, Y., Wang, J., \& Carranza, E. J. M. (2019). Deep learning and its application in geochemical mapping. In Earth-Science Reviews (Vol. 192, pp. 1-14). https://doi. org/10.1016/j.earscirev.2019.02.023 